\title{
Growth and postgrowth rapid thermal annealing of InAsN/InGaAs single quantum well on InP grown by gas source molecular beam epitaxy
}

\author{
Jyh-Shyang Wang ${ }^{\text {a) }}$ and Hao-Hsiung Lin \\ Room 419, Department of Electrical Engineering, National Taiwan University, Taipei, Taiwan, \\ Republic of China
}

(Received 21 May 1999; accepted 2 August 1999)

\begin{abstract}
InAsN/InGaAs single quantum wells (SQWs) with different nitrogen concentration have been successfully grown on InP substrates by gas source molecular beam epitaxy used rf plasma nitrogen source. Photoluminescence (PL) results of the as-grown samples show red-shifted PL peak energy and rapidly degraded intensity as the nitrogen concentration increases. The roughly estimated maximum nitrogen mole fraction in these samples is $0.4 \%$. Both the PL intensity and linewith of these InAsN/InGaAs SQWs were significantly improved after postgrowth rapid thermal annealing with the optimum temperature at $525-550{ }^{\circ} \mathrm{C}$ for samples with different nitrogen content. The improvement on $10 \mathrm{~K}$ PL intensity can be as high as 230 times, and the room temperature PL intensities of the annealed InAsN SQWs have been comparable to those of InAs SQWs used for laser diodes. Quantum well intermixing (QWI) induced blue-shifted PL spectra were also observed in these samples. The QWI threshold temperature decreases as the nitrogen concentration increases, which indicates that defects created by nitrogen incorporation may enhance the QWI. (C) 1999 American Vacuum Society. [S0734-211X(99)08205-0]
\end{abstract}

\section{INTRODUCTION}

Low nitrogen content zincblende III-V alloys have received much attention in the past few years. ${ }^{1-3}$ The large difference in atomic sizes and electronegativities of $\mathrm{N}$ and As has motivated the theoretical approach to understand the huge bowing parameter and also to ascertain the semiconductor or semimetal nature of these alloys. ${ }^{4-6}$ In addition, the large conduction band offset of these materials is very promising to overcome the poor temperature characteristics of conventional InGaAsP/InP system long-wavelength laser diodes. Recently, Kondow et al. reported InGaAsN grown on a GaAs substrate for a light-emitting material having a band gap suitable for long-wavelength laser diodes at $1.3 \mu \mathrm{m}^{2}$. Tu et al. also demonstrated InNAsP grown on a InP substrate for $1.55 \mu \mathrm{m}$ laser diodes. ${ }^{3}$ Both alloys are very important for an optical communication system.

In the midinfrared 2-5 $\mu \mathrm{m}$ wavelengths, InAsN alloy could also be a very promising material. Recently, we demonstrated high-quality $2.2 \mu \mathrm{m}$ InAs/InGaAs/InP highly strained multiquantum well lasers grown by gas-source molecular beam epitaxy (GSMBE). ${ }^{7}$ Using InAsN to replace InAs can ease the design limitation on quantum well thickness and decrease the band gap of quantum well. It reveals the possibilities of pushing laser emission to longer wavelength. However, there are still no definitive results on the growth of InAsN alloy. Beresford et al. had studied these alloys grown by plasma-source molecular beam epitaxy. ${ }^{8}$ They reported that InAsN alloy phase is metastable and forms in a fairly narrow temperature window of approximately $450-500{ }^{\circ} \mathrm{C}$. At higher temperatures or higher group $\mathrm{V}$ supersaturations, only separated phases of InAs and $\mathrm{InN}$ are observed. So far, there is still not any optical property

${ }^{a)}$ Electronic mail: jswang@crystal.ee.ntu.edu.tw data available. On the other hand, postgrowth thermal annealing is used to improve the quality of low nitrogen content III-V alloys. Rao et al. ${ }^{9}$ and Francoeur et al. ${ }^{10}$ had studied the postgrowth thermal annealing in GaAsN bulk materials. In this article, we demonstrated the InAsN/InGaAs SQW grown on InP substrates by GSMBE and studied the influence of postgrowth rapid thermally annealing treatments on their photoluminescence properties.

\section{EXPERIMENT}

The samples were grown on semi-insulating (100) InP substrates using a VG V-80H GSMBE system. Element In, $\mathrm{Ga}$, and thermally cracked $\mathrm{AsH}_{3}$ and $\mathrm{PH}_{3}$ sources were used. An EPI UNI-bulb rf plasma source operated at a radio frequency of $13.56 \mathrm{MHz}$ was used to generate active $\mathrm{N}$ species or $\mathrm{H}$ species. The rf power for generating $\mathrm{N}$ species was 300 $\mathrm{W}$. After the thermal cleaning of the InP substrate at $500^{\circ} \mathrm{C}$ under $\mathrm{P}_{2}$ flux, the brightness mode $\mathrm{H}_{2}$ plasma was first ignited and a $0.1-\mu \mathrm{m}$-thick InP layer was then grown as a buffer layer at $450{ }^{\circ} \mathrm{C}$. Some reports indicated that atomic hydrogen can enhance the removing of surface oxide and passivate some impurities and defects. ${ }^{11,12}$ Then, the growth was interrupted and the substrate temperature was reduced to $400{ }^{\circ} \mathrm{C}$ for $\operatorname{InGaAs} / \mathrm{InAs}(\mathrm{N}) / \mathrm{InGaAs} \mathrm{SQW}$ growth. The SQW structure layer is comprised of two 100-nm-thick $\mathrm{In}_{0.53} \mathrm{Ga}_{0.47} \mathrm{As}$ barriers and a $30-\AA$-thick $\operatorname{InAs}(\mathrm{N})$ well. The growth rate for $\operatorname{InAs}(\mathrm{N})$ was $1 \mu \mathrm{m} / \mathrm{h}$. There were no interruption at the heterointerfaces of the SQW. The gas line of the rf plasma source was switched immediately to $\mathrm{N}_{2}$ gas when the growth of the first SQW interface was starting. During the gas switching, the plasma still maintained high brightness mode. The rf power was turned off when the growth of the SQW was finished. The beam equivalent pressure (BEP) of the $\mathrm{N}$ source during the growth of InAsN 
TABLE I. Beam equivalent pressure (BEP) of N sources during InAs(N) layer growth, $10 \mathrm{~K} 1 e-1 h h$ PL energy, maximum improvement magnitude of $10 \mathrm{~K} \mathrm{PL}$ integrated intensity and the temperature of occurring quantum well intermixing (QWI) during RTA process.

\begin{tabular}{ccccc}
\hline \hline $\begin{array}{c}\text { No. of } \\
\text { samples }\end{array}$ & $\begin{array}{c}\text { BEP of N Sources } \\
(\mathrm{mbar})\end{array}$ & $\begin{array}{c}\text { 10 K 1e }-1 \mathrm{hh} \mathrm{PL} \\
\text { energy }(\mathrm{eV})\end{array}$ & $\begin{array}{c}\text { Maximum improvement } \\
\text { magnitude of 10 K PL } \\
\text { integrated intensity (times) }\end{array}$ & $\begin{array}{c}\text { Temperature of } \\
\text { occurring QWI }\left({ }^{\circ} \mathrm{C}\right)\end{array}$ \\
\hline $\mathrm{A}$ & 0 & 0.634 & 1.5 & 650 \\
$\mathrm{~B}$ & $<5 \times 10^{-5}$ & 0.631 & 1.7 & 600 \\
$\mathrm{C}$ & $5 \times 10^{-5}$ & 0.604 & 230 & 575 \\
$\mathrm{D}$ & $6 \times 10^{-5}$ & 0.596 & 25 & 600 \\
$\mathrm{E}$ & $9 \times 10^{-5}$ & 0.575 & 5.7 & 575 \\
\hline \hline
\end{tabular}

SQW was around $10^{-5}$ to $10^{-4}$ mbar, depending on the nitrogen flow rates. The BEP data of samples are summarized in Table I. Finally, a $0.1-\mu$ m-thick InP cap layer was overgrown on the SQW.

The annealing was performed using a ULVAC D91906 rapid thermal annealer. Before annealing, a spin-on silica capping layer was coated on the samples and baked at $400{ }^{\circ} \mathrm{C}$ for $30 \mathrm{~min}$ in air so as to prevent the desorption of the group $\mathrm{V}$ elements. We fixed the annealing time at $15 \mathrm{~min}$ and changed the annealing temperature from 500 to $650{ }^{\circ} \mathrm{C}$. Typical temperature fluctuation of this annealer was within $10^{\circ} \mathrm{C}$. The optical quality was evaluated using PL measurements. The $488 \mathrm{~nm}$ line of an argon laser was used as the excitation source and a closed cycle helium cryostat was used for low temperature measurements. The signal was detected using a liquid nitrogen cooled $\mathrm{InSb}$ photodiode with a Hamamatsu P3357-02 preamplifier. X-ray spectrum measurements were performed using Bede QC1A double crystal $\mathrm{x}$-ray diffractometer.

\section{RESULTS AND DISCUSSION}

The $10 \mathrm{~K} \mathrm{PL}$ spectra of the as-grown samples are shown in Fig. 1(a). As can be seen, the PL peak energy decreases with increasing nitrogen flux. This result indeed confirms the bowing effect due to the incorporation of nitrogen. However, the PL intensity degrades very rapidly with the increasing of nitrogen concentration, and the full width at half maximum (FWHM) also has similar behavior. Figure 1(b) shows the room temperature PL spectra of these as-grown samples. No room temperature luminescence can be detected in our PL system for samples C, D, and E. These results indicate the presence of high concentration nonradiative centers. Since there is a very large atomic-size difference between $\mathrm{N}$ and As ( 0.75 vs $1.20 \AA)$, the large local strain from the nitrogen incorporation in InAs crystal could result in inferior crystallinity. However, it could also be partially caused by the low growth temperature. In general, the more the $\mathrm{N}$ atoms incorporated into InAs, the worse the sample crystallinity, and the weaker the PL intensity. All of the behaviors are similar to those reported previously for the (In)GaAsN system. ${ }^{1-3,8-10,13,14}$ X-ray diffraction (XRD) spectra of these samples were also measured. However, the 30 - $\AA$-thick $\operatorname{InAs}(\mathrm{N})$ quantum well is too thin to make a difference be- tween the spectra of the samples with and without nitrogen. XRD simulation results also support this point.

The $10 \mathrm{~K}$ PL spectra of InAs SQW, sample A, before and after annealing for $15 \mathrm{~min}$ from 500 to $650^{\circ} \mathrm{C}$ are illustrated in Fig. 2(a). As can be seen, the postgrowth annealing leads to higher PL intensity and sharper FWHM. The PL peak position $(\sim 0.63 \mathrm{eV})$ shows only a slight blue-shift below $650{ }^{\circ} \mathrm{C}$. As the annealing temperature rises to $650{ }^{\circ} \mathrm{C}$, the $\mathrm{PL}$ peak position displays a significant blue-shift and the FWHM also increases. This is due to quantum well intermixing (QWI). ${ }^{15-17}$ The Ga atoms from the barriers diffuse into QW and increase the band gap energy of QW. The integrated PL intensity and FWHM are plotted against the annealing temperature in Fig. 2(b). Basically, the increase of the annealing temperature only results in slight improvements on the PL properties. The optimum temperature is around 600$625^{\circ} \mathrm{C}$. Because of the good as-grown quality of sample A, the improvement is not remarkable.
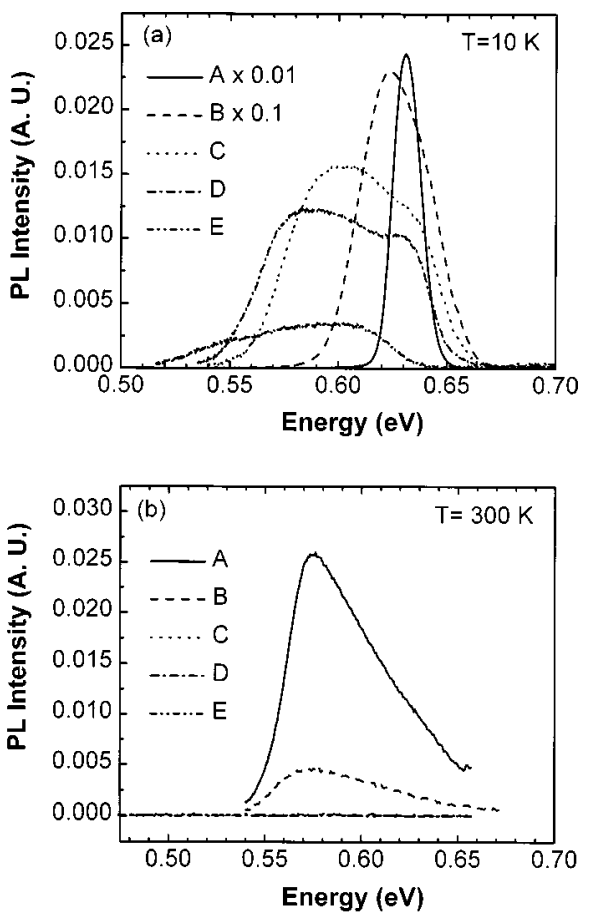

FIG. 1. PL spectra of as-grown $\operatorname{InAs}(\mathrm{N}) / \mathrm{InGaAs} \mathrm{SQW}$ at $10 \mathrm{~K}$ (a) and at room temperature (b). 

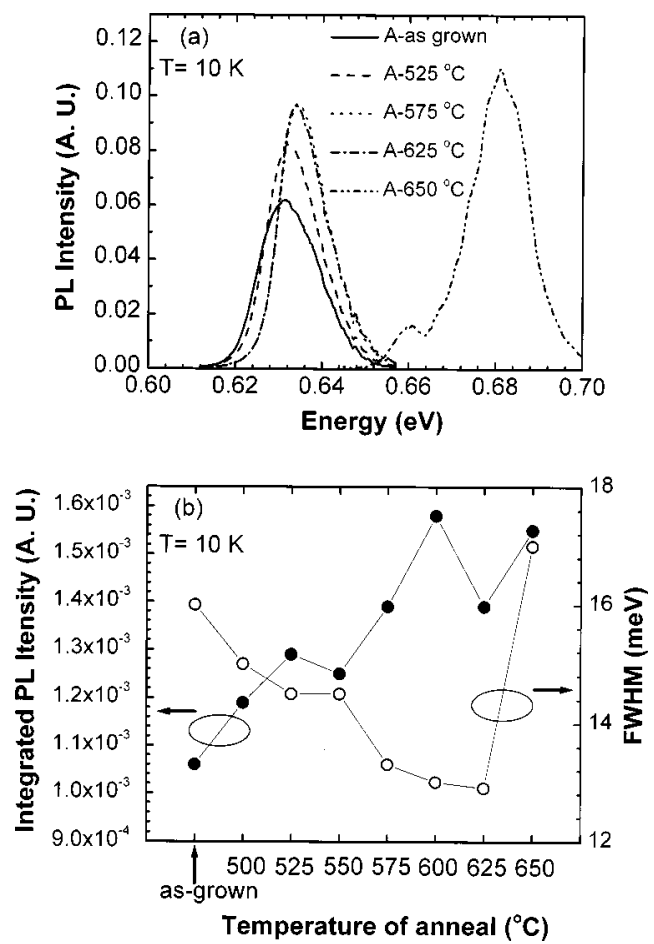

FIG. 2. (a) Low-temperature PL spectra of InAs SQW, sample A, before and after annealing at different temperatures for $15 \mathrm{~min}$. (b) Integrated PL intensity and FWHM as a function of annealing temperature.

On the other hand, there are very different behaviors for InAsN SQW samples with the same postgrowth annealing process. Figure 3(a) shows the $10 \mathrm{~K}$ PL spectra of sample E before and after 15 min annealing with different temperatures from 500 to $575^{\circ} \mathrm{C}$. As can be seen in Fig. 3(a), the postgrowth annealing leads to higher PL intensity and sharper FWHM. The improvement is very significant compared with the pure InAs SQW, i.e., sample A. The PL peak position $(\sim 0.57 \mathrm{eV})$ also shows almost no shift for the annealing below $575^{\circ} \mathrm{C}$. As the annealing temperature rises to $575^{\circ} \mathrm{C}$, a noticeable blue-shift on the PL peak energy is observed. The PL peak intensity also degrades significantly, even smaller than that of the as-grown sample. However, the FWHM does not increase. The integrated PL intensity and FWHM as functions of annealing temperature are plotted in Fig. 3(b). Below $575^{\circ} \mathrm{C}$, the increase of annealing temperature results in improvements both on the PL intensity and FWHM. The optimum temperature is around $525-550^{\circ} \mathrm{C}$. Other InAsN SQW samples, i.e., samples B, C, and D, all show very similar behaviors as those of sample E. Among them, sample $\mathrm{C}$ has the most significant improvement on 10 K PL intensity. The integrated intensity after annealing is about 230 times of that of before annealing. The detailed results of these samples are summarized in Table I. These results indicate that postgrowth annealing can effectively reduce the number of the nonradiative defects in the InAsN SQWs.

Compared with the pure InAs SQW, i.e., sample A, the red-shift of sample $\mathrm{E}$ due to nitrogen incorporation is about $60 \mathrm{meV}$. Kondow et al. reported that the band gap shrinkage
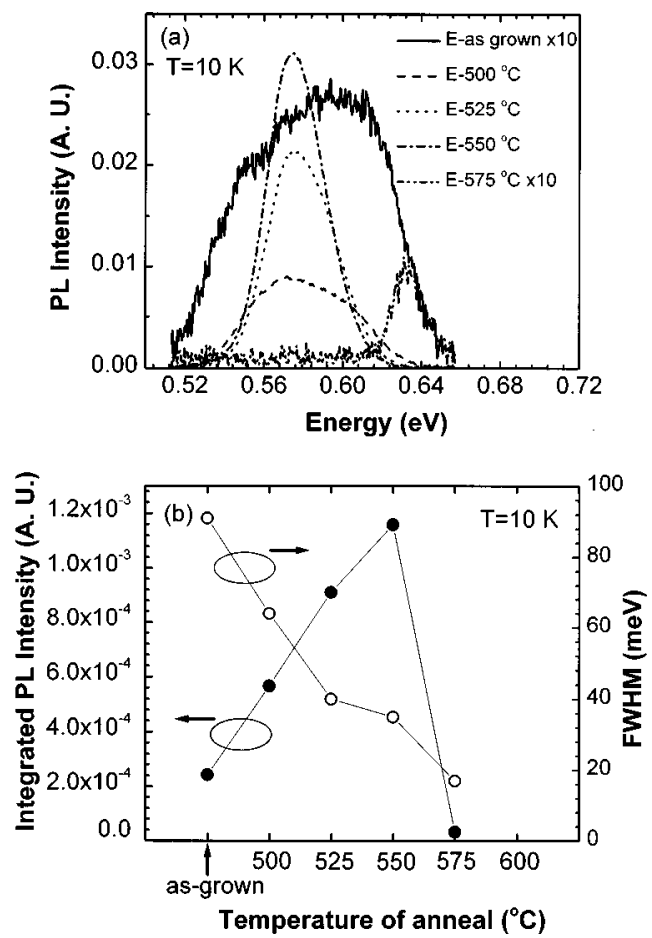

FIG. 3. (a) Low-temperature PL spectra of InAsN SQW, sample E, before and after annealing at different temperature for $15 \mathrm{~min}$. (b) Integrated PL intensity and FWHM as a function of annealing temperature.

coefficient of strained InGaAsN is $150 \mathrm{meV}$ per nitrogen concentration in percent. ${ }^{2}$ Francoeur et al. reported that the band gap reduction of GaAsN with a nitrogen mole fraction of $\sim 3 \%$ is $400 \mathrm{meV} .{ }^{10}$ According to these experimental results, the roughly estimated nitrogen mole fraction of sample $\mathrm{E}$ is around $0.4 \%$.

The threshold temperature of QWI decreases when the nitrogen concentration increases, as can be seen in Table I. Basically, the incorporation of nitrogen atoms into the InAs quantum well deteriorates the crystallinity and increases the density of defects. These defects may enhance the QWI and

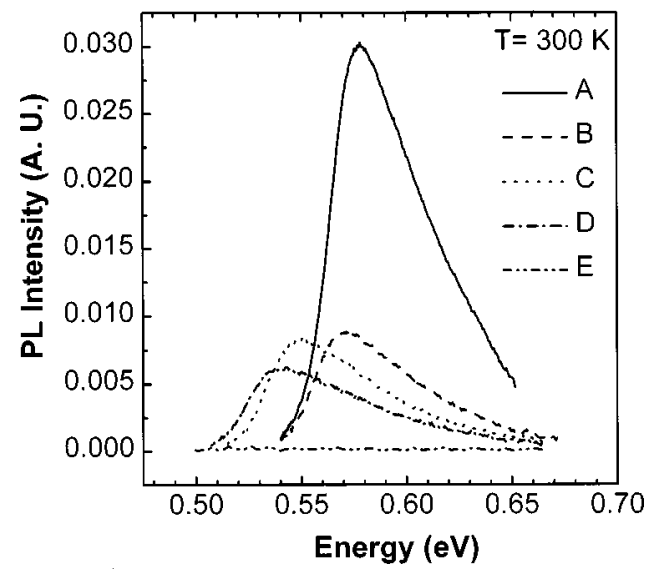

FIG. 4. Room temperature PL spectra of all InAs(N) SQW samples after annealing at their optimum temperatures. 
decrease the threshold temperature, which is consistent with the previous QWI studies on other compound alloys. ${ }^{18}$

Figure 4 shows the room temperature PL spectra of all InAs(N) SQW samples after annealing at their optimum temperature. As can be seen, the PL intensity of InAsN SQWs are improved after the postgrowth annealing. The after annealing PL intensities of samples C and D have especially been comparable with that of sample $\mathrm{B}$ and their peak intensities are just one-third of that of sample A, the InAs SQW, whose intensity is very closed to those of our previous laser devices. ${ }^{7}$ Notice that the room temperature luminescence of samples $\mathrm{C}$ and D are not observable before annealing, as can be seen in Fig. 1(b). Furthermore, the temperature dependence PL spectra of all samples have the same behaviors and show only one peak. This result indicates again that the emission is from the direct recombination across the band gap but not due to the deep level of InAs.

\section{CONCLUSION}

In summary, we have successfully grown InAsN/InGaAs SQW on InP substrates by GSMBE using a rf plasma nitrogen source. The poor as-grown optical quality of InAsN SQWs can be significantly improved with postgrowth annealing, and the optimum annealing temperature is about $525-550{ }^{\circ} \mathrm{C}$ for samples with various nitrogen contents. According to the red-shifted energy of the PL emission peak, the roughly estimated maximum nitrogen concentration is around $0.4 \%$. The defects enhance the QWI and reduce the threshold temperature of QWI. Combining the optimum growth condition and postgrowth annealing, InAsN SQWs with device quality have been obtained.

\section{ACKNOWLEDGMENT}

This work was supported by the National Science Council of the Republic of China under Contract No. NSC 87-2215E-002-010.

${ }^{1}$ M. Kondow, K. Uomi, T. Kitatani, S. Watahiki, and Y. Yazawa, J. Cryst. Growth 164, 175 (1996).

${ }^{2}$ M. Kondow, T. Kitatani, S. Nakatsuka, M. C. Larson, K. Nakahara, Y. Yazawa, M. Okai, and K. Uomi, IEEE J. Sel. Top. Quantum Electron. 3, 719 (1997).

${ }^{3}$ W. G. Bi and C. W. Tu, Appl. Phys. Lett. 72, 1161 (1998).

${ }^{4}$ S. Sakai, Y. Ueta, and Y. Teauchi, Jpn. J. Appl. Phys., Part 1 32, 4413 (1993).

${ }^{5}$ S. H. Wei and A. Zunger, Phys. Rev. Lett. 76, 664 (1996).

${ }^{6}$ L. Bellaiche, S. H. Wei, and A. Zunger, Phys. Rev. B 54, 17568 (1996).

${ }^{7}$ J. S. Wang, H. H. Lin, and L. W. Sung, IEEE J. Quantum Electron. 34, 1959 (1998).

${ }^{8}$ R. Beresford, K. S. Stevens, and A. F. Schwartzman, J. Vac. Sci. Technol. B 16, 1293 (1998).

${ }^{9}$ E. V. K. Rao, A. Ougazzaden, Y. Le Bellego, and M. Juhel, Appl. Phys. Lett. 72, 1409 (1998).

${ }^{10}$ S. Francoeur, G. Sivaraman, Y. Qiu, S. Nikishin, and H. Temkin, Appl. Phys. Lett. 72, 1857 (1998).

${ }^{11}$ K. D. Choquette, M. Hong, S. N. G. Chu, H. S. Luftman, J. P. Mannaerts, R. C. Wetzel, and R. S. Freund, Appl. Phys. Lett. 62, 735 (1993).

${ }^{12}$ M. Gal, A. Tavendale, M. J. Johnson, and B. F. Usher, J. Appl. Phys. 66, 968 (1989).

${ }^{13}$ H. P. Xin and C. W. Tu, Appl. Phys. Lett. 72, 2442 (1998).

${ }^{14}$ M. R. Gokhale, J. Wei, H. Wang, and S. R. Forrest, Appl. Phys. Lett. 74, 1287 (1999).

${ }^{15}$ A. S. Helmy, J. S. Aitchison, and J. H. Marsh, Appl. Phys. Lett. 71, 2998 (1997).

${ }^{16}$ O. P. Kowalski, C. J. Hamilton, S. D. McDougall, J. H. Marsh, A. C. Bryce, R. M. De La Rue, B. Vögele, C. R. Stanley, C. C. Button, and J. S. Roberts, Appl. Phys. Lett. 72, 581 (1998).

${ }^{17}$ G. Li, S. J. Chua, S. J. Xu, X. C. Wang, A. S. Helmy, M. L. Ke, and J. H. Marsh, Appl. Phys. Lett. 73, 3393 (1998).

${ }^{18}$ B. S. Ooi, Y. S. Tang, A. S. Helmy, A. C. Bryce, J. H. Marsh, M. Paquette, and J. Beauvais, J. Appl. Phys. 83, 4526 (1998). 